\title{
Seroprevalencia de Coxiella burnetii en trabajadores de fincas ganaderas del departamento de Antioquia
}

\section{Seroprevalence of Coxiella Burnetii in cattle farm workers in the department of Antioquia}

\author{
Carlos Alberto Betancur, Manuela Rubio, Juliana Barrera, \\ Juan Carlos Bedoya - Medellín (Colombia)
}

\section{Resumen}

Introducción: Coxiella burnetii es el agente etiológico de la fiebre Q, zoonosis asociada principalmente al contacto con ganado bovino y caprino. El principal modo de transmisión es el contacto con productos del parto, sangre, leche, lana, además de la inhalación de las esporas de la bacteria, que permite que se produzca infección aun en sitios alejados del reservorio.

Objetivo: describir la seroprevalencia de $C$. burnetii en una población de riesgo como los trabajadores de fincas ganaderas del departamento de Antioquia 2011-2012.

Metodología: se determinaron los niveles de anticuerpos $\mathrm{IgG}$, por inmunofluorescencia, determinantes del contacto previo con C. burnetti, en 102 trabajadores de fincas ganaderas localizadas en tres municipios del departamento de Antioquia.

Resultados: se encontraron $15(14.70 \%)$ muestras positivas para Ig G (fase I y II) contra Coxiella burnetii en un grupo de 92 hombres y 10 mujeres, sin que se estableciera relación entre el género y su seropositividad ( $\mathrm{p}=0.167$ ), edad (p: 0.889) y tiempo de exposición (p: 0.118).

Conclusión: la infección por $C$. burnetti es una zoonosis de importancia en nuestro medio y debe pensarse en ella al momento de enfrentarse a cuadros febriles tanto agudos como crónicos, en poblaciones de riesgo. (Acta Med Colomb 2015; 40: 20-23).

Palabras clave: Coxiella, fiebre $Q$, zoonosis.

\section{Abstract}

Introduction: Coxiella burnetii is the etiologic agent of Q fever, zoonosis mainly associated to contact with cattle and goats. The main mode of transmission is contact with products of delivery, blood, milk, wool, in addition to inhalation of spores of the bacterium, that allows to occur the infection even in places far from the reservoir.

Objective: to describe the seroprevalence of $C$. burnetii in a population at risk such as farm workers in Antioquia department 2011-2012.

Methodology: IgG antibody levels were determined by immunofluorescence, determinants of previous contact with $C$. burnetii in 102 workers of farms localized in three municipalities of Antioquia.

Results: 15 (14.70\%) samples positive for IgG (phase I and II) were found against Coxiella burnetii in a group of 92 men and 10 women, with no relationship between gender and seropositivity $(\mathrm{p}=0.167)$, age $(\mathrm{p}=0.889)$ and exposure time $(\mathrm{p}=0.118)$ established.

Conclusion: C. burnetii infection is a zoonosis of importance in our environment and should be thought of when faced with febrile pictures both acute and chronic, in populations at risk. (Acta Med Colomb 2015; 40: 20-23).

Key words: Coxiella, Qfever, zoonosis.
Dr. Carlos Alberto Betancur Jiménez: Internista Clínica Soma Medellín, Profesor Titular Universidad CES; Dras. Manuela Rubio Rivera y Juliana Barrera Correa: Egresadas Facultad de Medicina Universidad CES; Dr. Juan Carlos Bedoya Blandón: Bacteriólogo, MSc Docente Facultad de Medicina Universidad CES. Medellín (Colombia).

Correspondencia. Dr. Carlos A. Betancur J. Clínica Soma. Medellín (Colombia).

E-mail: cbetancurmed@gmail.com

Recibido: 16/II/2014 Aceptado: 14/X/2014 


\section{Introducción}

Coxiella burnetii es una bacteria (cocobacilo) intracelular obligada formadora de esporas causante de la fiebre Q, zoonosis que es transmitida a los humanos por aerosoles al contacto directo con animales infectados o con sus productos como leche, orina, heces, placenta, siendo esta última donde mayor concentración alcanza el microorganismo. Sus esporas que se aerosolizan, son altamente resistentes a la desecación y a la luz, pudiendo llegar a largas distancias y al ser inhaladas por el hombre producen la enfermedad (1-4). Se estima que se excretan $10^{9}$ microorganismos por gramo de tejido placentario de un animal infectado (5).

Los reservorios animales más frecuentemente asociados son el ganado bovino y caprino, pero también se han descrito en animales domésticos como gatos, perros y roedores. En Alemania se halló una seroprevalencia de $13 \%$ en perros y $26 \%$ en gatos (6). La infección en los animales puede darse por la picadura de garrapatas (2). Raros casos de transmisión persona-persona han sido descritos (7).

La fiebre Q es una zoonosis con distribución mundial, más que todo prevalente en zonas geográficas ganaderas ( 8 , 9), y se constituye también como enfermedad ocupacional (10), está altamente relacionada con el tiempo de exposición al reservorio animal, siendo mayor la seroprevalencia en granjeros, manipuladores de ganado y veterinarios que en la población general $(10,11)$. Se presenta como brotes epidémicos autolimitados o casos esporádicos, el mayor brote se presentó en Alemania entre los años 2007 y 2010 con 4026 personas afectadas (12-14).

En Holanda, la seroprevalencia de la población general es de $2.4 \%$, siendo en donadores de sangre hasta el $24 \%$ y en grupos de riesgo hasta el $83.8 \%$ (veterinarios), en Francia y Alemania los donantes tienen una seroprevalencia de 4 y $22 \%(15,16)$.

En Latinoamérica, los estudios relacionan también la seroprevalencia como enfermedad emergente y de riesgo ocupacional: en México hay áreas donde ésta es de 11\% y (17) y en la selva amazónica ecuatoriana se plantea la fiebre Q como causa frecuente de síndrome febril (18). La seroprevalencia en trabajadores de granjas en Trinidad es cercana a 5\% (19).

En Colombia se ha descrito desde el 2006 con seroprevalencia de $23.6 \%$ (9) y de $6.1 \%$ (20) yluego como agente etiológico de neumonía (21) y de endocarditis (22), relacionándola también como riesgo ocupacional.

En los animales, la infección cursa subclínica, o se detecta casualmente en abortos o malformaciones del feto por PCR o presencia del agente etiológico $(15,23,24)$. En el hombre, la enfermedad suele presentarse como un cuadro febril bizarro, síndrome seudogripal, neumonía atípica, o hepatitis; puede autolimitarse y pasar desapercibido o evolucionar a fase crónica, lo cual ocurre en $2 \%$ de los casos, y puede manifestarse como endocarditis, síndrome febril prolongado, meningoencefalitis, polineurorraiculitis, artritis, entre otras $(21,22,25)$.

En un brote de la enfermedad en Suiza, donde se confirmaron por serología 415 casos, 54\% cursaron sintomáticos y $2 \%$ requirieron hospitalización (26). La principal prueba diagnóstica para infección por $C$. burnetii es la serología $(27,28)$.

Se realizó un estudio de tipo descriptivo transversal, determinando la seroprevalencia de $C$. burnetii en trabajadores de fincas ganaderas de tres municipios de Antioquia, con el objetivo de llamar la atención acerca de la presencia de este germen en nuestro medio, que puede ser causante de enfermedad en la población general, principalmente en personas con factores de riesgo como son los trabajadores de la industria ganadera.

\section{Material y métodos}

El presente trabajo fue aprobado por el comité institucional de ética de la Universidad CES de Medellín, como consta en el acta número 35 del año 2010.

Se realizó un estudio descriptivo de corte transversal. Durante el periodo comprendido entre junio de 2011 y noviembre de 2012, por medio de un muestreo por conveniencia, se recolectaron 102 muestras de suero de trabajadores de fincas ganaderas localizadas en tres municipios antioqueños $(\mathrm{La}$ Ceja, Envigado y Caracolí). Todos los participantes aceptaron estar en el estudio firmando previamente el consentimiento informado. Se incluyeron trabajadores mayores de 18 años, y que llevaran un año o más de contacto con ganado o sus productos. A todos se les hizo una encuesta sociodemográfica.

Las muestras de sangre se recolectaron en tubos sin anticoagulante y el suero se obtuvo por centrifugación, y posteriormente se congeló a $-20^{\circ} \mathrm{C}$ hasta el momento de procesarlo en el laboratorio de la universidad CES.

Para la prueba serológica se utilizó un estuche comercial de la casa FOCUS diagnostics (Cypress, California, U.S.A.) que es un inmunoensayo fluorescente indirecto (IFA), para la detección de anticuerpos IgG humanos contra $C$. burnetii en suero humano. La sensibilidad y especificidad relativa de la prueba fue estimada en 100 y $99 \%$ respectivamente (comparado con un IFA de referencia y a una prueba de fijación del complemento de un laboratorio de referencia). La lectura de las placas se realizó mediante microscopio de fluorescencia y los resultados se interpretaron siguiendo las instrucciones del fabricante así:

\section{Detección de anticuerpos IgG humanos contra Coxiella burnetii}

Cada celda en la que se procesa la muestra del paciente, contiene dos áreas individuales para el antígeno: un área de antígeno de $C$. burnetii inactivado fase I y otro de antígeno de $C$. burnetii inactivado fase II.

\section{Interpretación de resultados: reactividad con ambos antígenos de Fase I y Fase II}

Positivo: $\geq 1: 16$ con ambos antígenos de fase I y fase II.

Fase II > Fase I: infección aguda

Fase II $\leq$ fase I: infección crónica.

Negativo: <1:16 con ambos antígenos fase I y fase II. 
Reactividad sólo con antígeno fase II >256: infección activa o reciente.

\section{Resultados}

Los resultados se resumen en la Tabla 1. Se les hizo un muestreo a 102 pacientes (92 hombres y 10 mujeres), y en 15 de ellos $(14,7 \%)$ se detectaron anticuerpos IgG contra $C$. burnetii (a una dilución $\geq 1: 16$, tanto fase I como fase II. En todos ellos los títulos de anticuerpos fase I fueron mayores que los de fase II). La edad fluctuó entre 18 y 73 años, con mediana de 42 años, sin que se estableciera relación entre esta variable y la seropositividad ( $\mathrm{p}=0.889$ ). El tiempo de exposición a ganado vacuno o sus productos, varió entre 1 y 66 años, relacionando el mayor tiempo de contacto con tener IgG positiva para C. burnetii, pero no de manera estadísticamente significativa $(\mathrm{p}=0.118)$.

El principal antecedente relacionado (aunque no estadísticamente significativo) con la seropositividad fue la fiebre durante el último año $(\mathrm{p}=0.112)$. Otras variables como el género y el sitio de procedencia se asociaron de forma no significativa con la seropositividad (valores de $\mathrm{p}=0.167 \mathrm{y}$ 0.097 respectivamente).

No hubo asociación significativa entre seropositividad y otras variables determinadas mediante encuesta, como consumo de leche cruda ( $\mathrm{p}=0.414)$ y contacto con productos del parto $(\mathrm{p}=0.212)($ Tabla 1$)$.

\section{Discusión}

Se halló una seroprevalencia para C. burnetii del $14.7 \%$ en las 102 muestras analizadas (10 mujeres y 92 hombres), con una mediana de edad de 42 años, siendo un valor intermedio entre los ya encontrados en nuestro país del 6.1 y $23.6 \%$ $(9,20)$, e inferiores a países con mayor prevalencia como Holanda, que en grupos de riesgo alcanza una seroprevalencia de hasta $83.8 \%(15,16)$; pero mayor o semejante a lo descrito para población general (bancos de sangre) en otros países como Francia, Holanda, Alemania y España de 4, 24, 22 y $23.15 \%$ respectivamente $(15,29)$.

La infección por Coxiella burnetii es subdiagnosticada en nuestro medio y esto es explicable por el desconocimiento de dicho germen como causante de enfermedad. En Latinoamérica hay estudios de seroprevalencia como en México de $11 \%$, Ecuador $4 \%$ y Trinidad 5\% (17-19). En los reportes colombianos el doctor Salim Mattar halló una seroprevalencia de 23.6\% en el área rural del departamento de Córdoba y en diferentes regiones del departamento de Antioquia. Molina et al describieron una seroprevalencia de $6.1 \%(9,20)$. En una investigación realizada en el área urbana de Medellín sobre la etiología de la neumonía adquirida en la comunidad, encontramos $C$. burnetii como agente causante en 5.4\% (17 pacientes) (21), posteriormente se describió el primer caso de endocarditis por este microrganismo en el año 2012 (22), hechos que muestran la presencia de la enfermedad en nuestro medio y la necesidad de tenerla en cuenta en el estudio de síndromes febriles. En la presente investigación hallamos una seroprevalencia de $14.7 \%$, que está en un punto intermedio en lo descrito en Colombia y por debajo de países de otras latitudes con un alto riesgo ocupacional.

Está evidenciada la asociación entre la seroprevalencia y factores de riesgo. Si bien nosotros no encontramos una relación estadísticamente significativa, los pacientes expuestos a los productos del parto presentaron seropre-

Tabla 1. Relación entre variables sociodemográficas determinadas por encuesta y presencia de anticuerpos IgG contra C. burnetii, en trabajadores de fincas ganaderas de tres municipios antioqueños.

\begin{tabular}{|c|c|c|c|c|c|c|c|}
\hline \multirow[t]{3}{*}{ Variable } & \multirow[t]{3}{*}{ Categoría } & \multicolumn{4}{|c|}{ Positivo } & \multirow{3}{*}{$\begin{array}{c}\text { Chi } \\
\text { cuadrado }\end{array}$} & \multirow{3}{*}{$\begin{array}{c}\text { Valor } \\
\mathbf{p}\end{array}$} \\
\hline & & \multicolumn{2}{|c|}{$\mathrm{Si}$} & \multicolumn{2}{|c|}{ No } & & \\
\hline & & $\mathbf{n}$ & $\%$ & $\mathbf{n}$ & $\%$ & & \\
\hline \multirow[t]{6}{*}{ Edad } & $12-24$ & 1 & 6.7 & 11 & 12.6 & \multirow[t]{6}{*}{1.696} & \multirow[t]{6}{*}{0.889} \\
\hline & $25-34$ & 3 & 20 & 18 & 20.7 & & \\
\hline & $35-44$ & 4 & 26.7 & 21 & 24.1 & & \\
\hline & $45-64$ & 4 & 26.7 & 20 & 23 & & \\
\hline & $55-64$ & 3 & 20 & 12 & 13.8 & & \\
\hline & 65 y más & 0 & 0 & 5 & 5.7 & & \\
\hline \multirow[t]{2}{*}{ Sexo } & Femenino & 0 & 0 & 10 & 11.5 & \multirow[t]{2}{*}{1.912} & \multirow[t]{2}{*}{0.167} \\
\hline & Masculino & 15 & 100 & 77 & 88.5 & & \\
\hline \multirow[t]{3}{*}{ Residencia } & Caracolí & 9 & 60 & 73 & 83.9 & \multirow[t]{3}{*}{4.657} & \multirow[t]{3}{*}{0.097} \\
\hline & Envigado & 4 & 26.7 & 9 & 10.3 & & \\
\hline & La Ceja & 2 & 13.3 & 5 & 5.7 & & \\
\hline \multirow{3}{*}{$\begin{array}{l}\text { Tiempo de exposición } \\
\text { (Años) }\end{array}$} & $1-10$ & 4 & 26.7 & 14 & 16.1 & \multirow[t]{3}{*}{4.271} & \multirow[t]{3}{*}{0.118} \\
\hline & $11-20$ & 1 & 6.7 & 28 & 32.2 & & \\
\hline & Más de 20 & 10 & 66.7 & 45 & 51.7 & & \\
\hline \multirow[t]{2}{*}{ Contacto parto } & Sí & 13 & 86.7 & 62 & 71.3 & \multirow[t]{2}{*}{1.559} & \multirow[t]{2}{*}{0.212} \\
\hline & No & 2 & 13.3 & 25 & 28.7 & & \\
\hline \multirow[t]{2}{*}{ Leche cruda } & Sí & 8 & 53.3 & 56 & 64.4 & \multirow[t]{2}{*}{0.666} & \multirow[t]{2}{*}{0.41} \\
\hline & No & 7 & 46.7 & 31 & 35.6 & & \\
\hline \multirow[t]{2}{*}{ Fiebre } & Sí & 4 & 26.7 & 42 & 48.8 & \multirow[t]{2}{*}{2.531} & \multirow[t]{2}{*}{0.112} \\
\hline & No & 11 & 73.3 & 44 & 51.2 & & \\
\hline
\end{tabular}


valencia mayor. Poblaciones de riesgo, como veterinarios, granjeros, trabajadores de la industria ganadera, y pastores, están expuestos al reservorio (ganado bovino) $(10,16,19$, $20)$, constituyéndose la fiebre $Q$ como una enfermedad ocupacional que exige tomar medidas de salud pública para evitar su transmisión en las fincas de producción bovina, implementando herramientas como reporte de casos, elementos de bioseguridad, desecho adecuado de los productos del parto animal, entre otros.

Hay necesidad de más estudios epidemiológicos en donde el tamaño de la muestra sea mayor, puedan considerarse otras variables epidemiológicas, se estudien otras regiones geográficas y se tenga también en cuenta a la población no expuesta al reservorio.

Se insiste en la importancia de la fiebre $Q$ como enfermedad prevalente en nuestro medio y por lo tanto debe ser considerada de manera importante en el diagnóstico diferencial de síndromes febriles agudos o prolongados, pues en zonas endémicas el diagnóstico se enfoca más en enfermedades como síndrome catarral, brucellosis, malaria, dengue y fiebre amarilla. Las secuelas de la enfermedad crónica son graves e incluso mortales, y pueden evitarse con un diagnóstico temprano y tratamiento antibiótico sencillo.

\section{Conclusiones}

- Hallamos una seroprevalencia de $14.7 \%$ de 102 trabajadores que están en contacto con ganado bovino.

- No hay asociación estadísticamente significativa entre las variables sociodemográficas evaluadas y la seropositividad.

- El tiempo de exposición (10/15 positivos con exposición mayor a 20 años), y el contacto con productos de parto (13/15 positivos), fueron las variables más relacionadas con la seropositividad.

- La infección por C. burnetti es una zoonosis de importancia en nuestro medio y debe pensarse en ella al momento de enfrentarse a cuadros febriles tanto agudos como crónicos en poblaciones de riesgo.

\section{Agradecimientos}

Los autores queremos expresar nuestros agradecimientos al área de gestión del conocimiento de la Universidad CES por la financiación de esta investigación, a la Dra. Bibiana Castro por el apoyo en el análisis estadístico y a la docente María Victoria Mejía por su apoyo en las pruebas de laboratorio.

\section{Referencias}

1. Tissot-Dupont H, Raoult D. Q fever. Infect Dis Clin 2002; 22: 505-514

2. Fariñas MTF, Collado CM. Infection by Coxiella burnetii (Q fever)]. Enferm Infecc Microbiol Clin 2010; 28 Suppl 1: 29-32.

3. Muñoz-Sanz A, Vera A, Rodriguez Vidigal FF. Q fever in Extremadura: an emerging infection. Enferm Infecc Microbiol Clin 2007; 25: 230-234

4. Ruiz-Fons F, Astobiza I, Barandika JF, Hurtado A, Atxaerandio R, Juste RA, Garcia-Perez AL. Seroepidemiological study of Q fever in domestic ruminants in semi-extensive grazing systems. BMC Vet Res. 2010: 6: 3

5. Arricau-Bouvery N, Souriau A, Lechopier P, Rodolakis A. Experimental Coxiella burnetii infection in pregnant goats: excretion routes. Vet Res 2003; 34: 423-33

6. Werth D, Schmeer N, Müller HP, Karo M, Krauss H. Demonstration of antibodies against Chlamydia psittaci and Coxiella burnetii in dogs and cats: comparison of the enzyme immunoassay, immunoperoxidase technic, complement fixation test and agar gel precipitation test. Zentralbl Veterinar Med B 1987; 34: 165-76.

7. Miceli MH, Veryser AK, Anderson AD, Hofinger D, Lee SA, Tancik C. A case of person-to-person transmission of $\mathrm{Q}$ fever from an active duty serviceman to his spouse. Vector Borne Zoonotic Dis 2010; 10: 539-541.

8. Kim JK, Hahn DY Lee MG, Jung KS, Ogawa M,Kishimoto T, Lee ME, Lee SJ. Seroprevalence of Coxiella burnetii Infection in dairy cattle and non-symptomatic people for routine health screening in Korea. J Korean Med Sci 2006; 21: 823-826.

9. Mattar S,Parra M. Detection of antibodies to Anaplasma, Bartonella and Coxiella in rural inhabitants of the caribbean area of Colombia. Rev MVZ Cordoba 2006: 11; 781-789.

10.Ellen AS, Whitney RF. Massung AJ, Candee EC, Ailes LM, Myers NE, Patterson RL, Berkelman. Seroepidemiologic and occupational risk survey for Coxiella burnetti antibodies among US veterinarians, CID 2009; 48: 550-555.

11. de Rooij MMT, Schimmer B, Versteeg B, Schneeberger P, Berends BR, Heederik D, van der Hoek W, Wouters IM. Risk Factors of Coxiella burnetii(Q Fever) Seropositivityin Veterinary Medicine Students. PLOS ONE 201; 7: e32108.

12. Van den Brom R, Vellema $P$. Q fever outbreaks in small ruminants and people in the Netherlands. Small Ruminant Res 2009; 86: 74-9.

13. Van der Hoek W, Dijstra F, Wijers N, Rietveld A, Wijkmans CJ, van Steenbergen JE, et al. Three years of Q fever in the Netherlands: faster diagnosis. Ned Tijdschr Geneeskd 2010; 154: A1845.

14. Schimmer B, Dijkstra F, Vellema P, Schneeberger PM, Hackert V, ter Schegget R, et al. Sustained intensive transmission of Q fever in the south of the Netherlands. EuroSurveillance 2009; 14(19): pii19210.

15. Georgiev M, Afonso A, Neubauer H, Howard NeedhamThiéry R, Rodolakis A, RoestHJ, Stärk KD, Stegema JA, Vellema P, van der Hoek W, More SJ. Q fever in humans and farm animals in four European countries, 1982 to 2010. Eurosurveillance 2013; 18: 1-13

16. Schimmer B, Lenferink A, Schneeberger P, Aangenend H, Vellema P, Hautvast $\mathbf{J}$, et al. Seroprevalence and risk factors for Coxiella burnetii ( $Q$ fever) seropositivity in dairy goat farmers' households in The Netherlands, 2009-2010. PLoS one. 2012; 7: e42364.

17. Araujo-Meléndez, Sifuentes J, Bobadilla M, Aguilar A, Torres O, Ramirez J, Ponce de Leon A, Ruiz G, Guerrero L.What do we know about Q fever in Mexico?. Revista de investigación clínica, 2012; 64: 541-545.

18. Manock SR,Jacobsen KH, de Bravo NB,Russell KL,Negrete M,Olson JG,Sanchez JL, Blair PJ,Smalligan R,Quist BK, FreireEspín J,Espinoza,Fiona ER,MacCormick,Fleming LC, Kochel T. Etiology of Acute Undifferentiated Febrile Illness in the Amazon Basin of Ecuador. Am J Trop Med Hyg 2009; 81:146-151

19. Adesiyun A, Dookeran S, Stewart-Johnson A, RahamanS, Bissessar S. Frequency of seropositivity for Coxiella burnetii immunoglobulins in livestock and abattoir workers in Trinidad. New Microbiologica 2011: 34; 219-224

20.LP, Rios S, Zapata R, Polanco D,Zapata M,Rios L,CardonaX,Giraldo LF,Lopera JA,Gutierrez LA. Evaluación de algunas zoonosis en productores ganaderos de la subregión norte del departamento de Antioquia, Colombia. Infectio 2012; 16 (S1): 60 .

21. Betancur CA, Vélez L, Rueda Z. Características clínicas de la neumonía adquirida en la comunidad asociada a bacterias atípicas y virus respiratorios. Act Med Col 2006; 31 sup: 326-327.

22. Betancur CA, Munera AG. Endocarditis por Coxiella burnetii. Fiebre Q. Act Med Col 2102; 37: 31-33

23. Sidi-Boumedine K, Rousset E, Henning K, Ziller M, Niemczuck K, Roest HIJ, et al. Development of harmonised schemes for the monitoring and reporting of Q Fever in animals in the European Union. EFSA 2010. 48 pp.

24. Yingst SL, Opaschaitat $P$, Kanitpun R, Thammasart S, Ekgatat M, Jirathanawat V, Wongwicharn P. Q fever surveillance in rumiants, Thailand 2012. Emerg Infect Dis 2013; 19: 2056-2058.

25. Bloju A, Balas R, Escobar M, Cerrada E, Rubio E. Fiebre Q. A propósito de tres formas de presentación. FMC 2011;18 :552-556.

26. Dupuis G, Vouilloz M. An important outbreak of human Q fever in a Swiss Alpine valley. Int J Epidemiol 1987; 16: 282-7

27. Thiele D, Karo M, Krauss H. Monoclonal antibody based capture ELISA/ELIFA for detection of Coxiella burnetii in clinical specimens. Eur. J. Epidemiol 1992; 8: $568-574$

28. Dorko E, Kalinova Z, Weissova T, Pilipcinec E. Seroprevalence of antibodies to Coxiella burnetii among employees of the Veterinary University in Kosice, eastern Slovakia. Ann Agric Environ Med 2008; 15: 119-124.

29. Bartolomé J,Riquelme E, Hernández-Pérez N, García-Ruiz S, Luján R, Lorente S, Medrano-Callejas $\mathbf{R}$, Crespo MD. Seroepidemiología de la infección por Coxiella burnetii en donantes de sangre en Albacea. Enferm Inf y Microb Clin 2007:25. 